\title{
TIME-RESOLVED SPECTROSCOPY AND DOPPLER TOMOGRAPHY OF UX UMa
}

\author{
V. F. Suleimanov, ${ }^{1}$ V. V. Neustroev ${ }^{2}$ N. V. Borisov ${ }^{3}$ and I. S. Fioktistova ${ }^{3}$
}

\begin{abstract}
Medium resolution spectroscopy of nova-like cataclysmic variable UX UMA was performed using the 6-m telescope SAO RAS in April 1999. Obtained spectra cover the total orbital period including eclipse phases and allow us to reproduce the radial velocity curve. The radial-velocity variations of the $\mathrm{H} \beta$ emission line are found to have semi-amplitude of about $100 \mathrm{~km} / \mathrm{s}$.
\end{abstract}

The nova-like cataclysmic variable UX UMA is well known short period $\left(0^{d} .1966713\right)$ eclipsing close binary. It has been extensively investigated in the past (Schlegel ot al. 1983, Shafter 1984, Rutten et al. 1994, Baptista et al. 1995, Knigge et al. 1998, Froning et al. 2003). The radial velocity curves for different emission and absorption lines with semiamplitudes $K_{1}$ ranged from 140 to $360 \mathrm{~km} / \mathrm{s}$ were obtained by Schlegel it al. (1983). Shafter (1984) found $\mathrm{K}_{1}$ for $\mathrm{H} \alpha$ equal $157 \pm 6 \mathrm{~km} / \mathrm{s}$ and this value is used now for UX UMA (sce, for example, Baptista et al. 1995). On the other hand, Froning et al. (2003) found the extremely low $K_{1}$ value $(70 \mathrm{~km} / \mathrm{s})$ for far ultraviolet absorption lines. Obtaining an accuracy value of $\mathrm{K}_{1}$ is one of the main aims of our time-resolved spectroscopy of UX UMA. A total of 63 medium resolution $(\sim 2.6 \AA)$ spectra of UX UMA in the wavelengtl range $3920-5250 \AA$ and with exposures of $300 \mathrm{~s}$ werc obtained on 6 -m telescope at Special Astrophysical Observatory during 1999 April 8 and 10. The spectra show $\mathrm{H} \beta, \mathrm{HeII} \lambda 4686$, CIII $\lambda 4648$ in emission for all phases (Fig. 1). $\mathrm{H} \delta, \mathrm{H} \epsilon$, HeI $\lambda 4471$ and MgII $\lambda 4481$ are in absorption at all phases except eclipse. Using the double-gaussian fitting method (Schneider \& Young 1980; Shafter 1983), we have determined $\mathrm{K}_{1}$ (from $\mathrm{H} \beta$ ) to be $100 \pm 20 \mathrm{~km} \mathrm{~s}^{-1}$. It means a white dwarf mass is greater than one solar mass, because the secondary mass $\left(0.45 \mathrm{M}_{\odot}\right)$ and the inclination angle $\left(i=72^{\circ}\right)$ are good known from the orbital period and the eclipse duration (Baptista et al. 1995; Shafter 1984). We have also found that during our observations UX UMA showed some fea-

\footnotetext{
${ }^{1}$ Department of Astronomy, Kazan State University: Kremlevskaya str. 18, 420008, Kazan, Russia (vals@ksu.ru).

${ }^{2}$ Computational Astrophysics Laboratory, National University of Ireland, Galway, Ireland (benj@it.nuigalway.ie).

${ }^{3}$ Special Astrophysical Observatory, Russia.
}

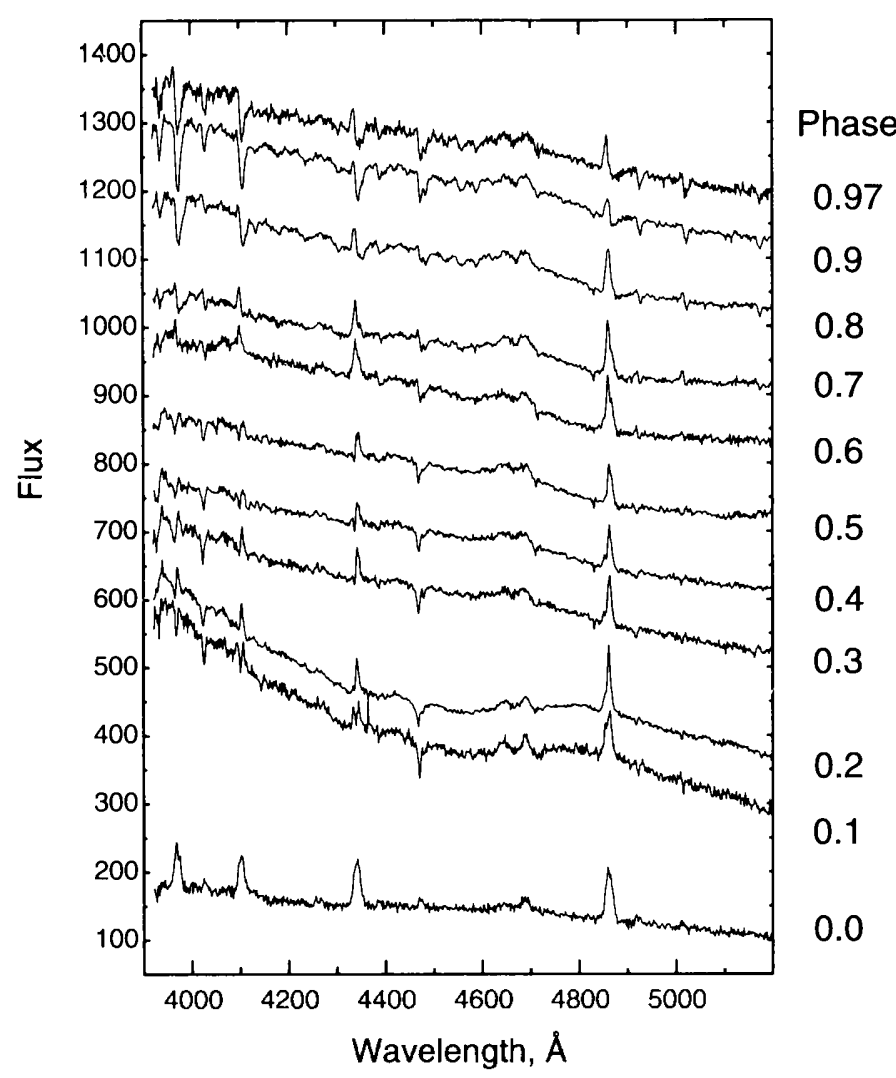

Fig. 1. The phase-resolved spectra of UX UMA. All spectra are shifted to each other except phases 0.0 and 0.1 .

tures which are commonly used for the identification of the SW Sex subclass stars. We have used Doppler tomography to investigate the emission line brightness distributions along the accretion disk surface.

V.S. acknowledges financial support from the Russian Foundation for Basic Research (grant 0217-174) and the Program of Support of Leading Scientific Schools of Russia (project 1789.2003.02).

\section{REFERENCES}

Baptista, R. et al., 1995, ApJ, 448, 395

Froning, C., Long, K., \& Knigge, C., 2003, ApJ, 584, 433

Knigge, C. et al., 1998, ApJ, 499, 414

Rutten, R. G. et al., 1994, A\&A, 283, 441

Schlegel, E., Honeycutt, R., \& Kaitchuck, R. 1983, ApJSS, 53, 397

Schneider. D., \& Young, P., 1980, ApJ, 238, 946

Shafter, A., 1983, ApJ 267. 222

Shafter, A., 1984, AJ, 89, 1555 